\title{
Proceeding
}

Supplementary Issue: Autumn Conferences of Sports Science. Costa Blanca Sports Science Events, 2-3 November 2018.

Alicante, Spain

\section{Effects of swimming and water walking on body composition and spirometric values in young children}

\author{
SAMUEL AA HONÓRIO1 ${ }^{1}$, PEDRO DUARTE MENDES MENDES ${ }^{1}$, MARCO BATISTA ${ }^{1}$, JOÃO \\ SERRANO ${ }^{1}$, RUI MIGUEL DUARTE ${ }^{1}$, JOAO OLIVEIRA², JOÃO PETRICA ${ }^{1}$ \\ ${ }^{1}$ SHERU (Sport, Health and Exercise Research Unit), Polytechnic Institute of Castelo Branco, Portugal \\ ${ }^{2}$ Polytechnic Institute of Castelo Branco, Portugal
}

\begin{abstract}
Aquatic activities have been recommended as frequent practices due to the physical properties of water with improvements in body composition of young. Objective: To study if there are differences in body composition and spirometric values in children who practice swimming complemented with water walking and those who only practice swimming. Methodology: 28 individuals (6 to 12 years) were divided into two groups: swimming group (SG: $n=9$ ) and swimming complemented with water walking group (SWWG: $n=19$ ) in three different moments with 6 weeks between them. For body composition a bio-impedance scale was used and an anthropometric tape for the waist circumference. For spirometric values: forced vital capacity (FVC), forced expiratory volume in 1 second (FEV1) and even peak expiratory flow (PEF) a Cosmed Microquark spirometer was used. For statistical procedures the SPSS (20.0) program for descriptive statistics, the Shapiro Wilk test for testing the normality, inferential statistics (non-parametric Mann-Whitney tests, Friedman's Anova), and for the effect size the d-Cohen test. Results: Regarding the inter-group analysis (comparison between the $S G$ and SWWG) we observed that there were significant differences in weight $(p=0,004)$, forced expiratory volume in 1 second (FEV1: $p=0,025$ ) and peak expiratory flow (PEF: $p=0,033$ ). Concerning intra-group differences (improvements in the SG and SWWG), the SWWG showed significant improvements in weight muscle mass $(p=0,029)$, fat mass $(p=0,002)$, percentage of water $(p=0,018)$, body mass index (BMl:

Corresponding author. SHERU (Sport, Health and Exercise Research Unit), Instituto Politécnico de Castelo Branco, Castelo Branco, Portugal.

E-mail: samuelhonorio@ipcb.pt

Supplementary Issue: Autumn Conferences of Sports Science. Costa Blanca Sports Science Events, 2-3 November 2018. Alicante, Spain.

JOURNAL OF HUMAN SPORT \& EXERCISE ISSN 1988-5202

(c) Faculty of Education. University of Alicante.

doi:10.14198/jhse.2019.14.Proc1.06
\end{abstract}


$p=0,000)$, body percentiles $(p=0,000)$, forced vital capacity (FVC: $p=0,003)$ and forced expiratory volume in 1 second (FEV1: $p=0,008$ ). We have concluded that the practice of swimming and water walking have benefits with differences in the analysed group variables, however, the two activities complemented (swimming and water walking) present improvements much more significant. Key words: Water; Body composition; Spirometric values.

\section{Cite this article as:}

Honório, S.A., Mendes, P.D., Batista, M., Serrano, J., Duarte, R.M., Oliveira, J., \& Petrica, J. (2019). Effects of swimming and water walking on body composition and spirometric values in young children. Journal of Human Sport and Exercise, 14(1proc), S47-S58. doi:https://doi.org/10.14198/jhse.2019.14.Proc1.06 


\section{INTRODUCTION}

According to Chu and Rhodes (2001) aquatic activities, due to the physical properties of water, especially the fluctuation, can be alternatives of exercises indicated for obese, by reducing the impact on the joints responsible for the weight support of the body. Within the aquatic activities, it is emphasized that walking or deep running, can be executed with or without using float equipment. In addition, the drag is higher in aquatic exercises due to the viscosity of water. Walking in water, in addition to the reduction of joint stress, provides a higher energy expenditure to overcome the resistance of the water (Keino, Born, \& Plasqui, 2014; Lopes, Bento, Lazzaroto, Rodacki \& Leite, 2015). We intend with this investigation to verify if this activity (water walking) brings health benefits to children, since there are several pathologies, namely the obesity and children sedentary lifestyle. Children no longer play, spend most of their days seated and indoors. According to Neto, Cordovil, \& Lopes (2015), nowadays, children have less capacity for coordination, less capacity for spatial perception, have less pleasure to use the body for effort, have the difficulty play in group, which causes them to sit for a long time. The playgrounds are often organized according to a work model, or a model of pedagogical functioning, which has more to do with the pedagogical learning required or considered useful, let alone with the activities of the body in movement. We chose this area because aquatic activities have some specific characteristics such as buoyancy, hydrostatic pressure and low impact on the joints of overweight people and increased energy expenditure. Water has several important characteristics such as viscosity and resistance to friction, which offers an increase in resistance to exercise, which naturally leads to increased energy expenditure of the body. Exercises in the aquatic environment are responsible for a 34\% increase in energy required during the same exercise if it is performed in the ground (Kruel, 2000). The present study seeks to know if there are significant differences between the two groups, swimming group that only practices swimming lessons (SG) and swimming with water walking group (SWWG) that practice swimming complemented with water walking and verified if there are significant improvements in each of the groups (SG and SWWG) at the end of the three evaluation moments. We had 28 children aged from 6 to 12 years. This work may also inspire other researchers to develop deeper into this line of research by applying this test to other populations of other age groups or possibly to other forms of evaluation.

According to Nahas (2013), physical fitness is directly related to health by focusing on components that aim at maintaining health, preventing and reducing disease risks, such as the capacity for cardiorespiratory endurance, musculoskeletal fitness and body composition. According to Kristensen, Moeller, Korsholm, Kolle, Wedderkopp, Froberg \& Andersen (2010) it is recognized that high levels of physical fitness are related to the prevention and combat of chronic degenerative diseases. Physical fitness is defined as a biological attribute for the ability to perform physical exertion. Also, physical activity should be understood as a multidimensional constructor in which it includes the intensity, duration and frequency of body movements (Bianchi, Augusto \& Keller, 2013). It is suggested by Faigenbaum (2015) that children and adolescents perform physical activity for at least 60 minutes with a moderate to vigorous intensity every day, but the young population does not reach this register. Excessive time spent in sedentary behaviours such as watching television, playing video games and other activities contribute to this problem (Troiano, Berringan, Dodd, Mâse, Tilert \& McDowell, 2008). Weekly physical activity routine at all ages does not only contribute to an improvement in physical appearance but also improves the decrease in body fat and body mass index (BMI). Practicing regular physical activity brings significant improvements in the health of these population. Several researchers indicate that the practice of physical activity at these ages contributes to a greater health-related fitness, improvements in preventing cardiovascular and metabolic diseases, a risk reduction of cardiovascular disease in adulthood, risk prevention of developing type 2 diabetes in childhood and adults, increased bone health and development, improvements in mental health and well-being, improvements in cognitive and academic performance, and improvements in motor control and physical functioning (Katzmarzyk et al. 
2014). Children and adolescents should practice a minimum of 60 minutes of moderate to vigorous physical activity at least 3 times a week. During the recommended exercise period of physical activity, muscle and bone strengthening activities should be included in the program at least 3 days a week. For strengthening muscle exercises with push-ups, climbing bars may be a good option. For moderate or vigorous activities, running, swimming or cycling are also excellent options. Physical activity is beneficial for cardiovascular and metabolic health, but physical activity always depends on intensity. Activities such as basketball, running and swimming are associated with greater benefits as these activities consume greater energy expenditure (Katzmarzyk et al. 2014). An active life brings health benefits, as the quality of life increases, anxiety and depression decrease, body fat is reduced, fat-free mass begins to manifest itself positively and pathologies such as diabetes, hypertension, cardiovascular diseases do not manifest in a significant way (Stella, Vilar, Lacroix, Fisberg, Santos, Mello \& Tufik, 2005).

Paulo, R. (2014) states that spirometry is an examination that helps in the diagnosis, prevention and quantification of ventilatory disorders, and can be performed during slow breathing or during a forced expiratory manoeuvred.

According to McGlone, Venn, Walters, \& Wood-Baker (2006) a reduction in forced expiratory volume in the first second (FEV1) is the most common outcome. However, the literature is controversial as to whether the reduction in physical activity level is directly linked to the degree of airflow limitation or whether other factors interfere. In addition, it is not known whether other spirometric variables, such as maximal voluntary ventilation and inspiratory capacity, are more closely related than FEV1 to the level of daily physical activity, as well as exercise capacity and respiratory muscle strength. The objective of this study was to investigate the relationship between physical activity in daily life and spirometric impairment assessed by different variables in patients with COPD and to investigate the relationship of these spirometric variables with functional exercise capacity and respiratory muscle strength. The same situation was observed in the Maclntyre (2009) where general physical activity was significantly associated with forced expiratory volume in one second. Sharifi, Mazreno, Salmani, \& Abyar (2014) show that the implementation of selected activities such as walking for 30-minute periods for eight weeks has a positive impact on spirometry parameters in children aged 5 to 10 years, as well as mobility. A program of physical exercises at the level of strengthening the diaphragm with the placement of light "free weights" in the abdominal area improve the symptoms in breathing caused by exercise, mental state and tolerance of the activity, an improvement of sports techniques in their activities daily and children's games. It is thus identified that the increase in the maximum amount of expiratory flow and expiratory volume force in the first seconds are directly related and that physical exercises do not always improve all spirometry parameters, but they are most effective.

The water walking exercise can be characterized by two types: walking in a shallow pool and walking in a deep pool (Dowzer \& Reilly, 1998; Barela, Stolf \& Duarte, 2006). The water walking can be incorporated into the main part or the final part in other aquatic programs. This activity is easily assimilated by the students and their choreography is quite simple and with easy instructions for those who teach this activity. In addition to be a physical benefit program, it also encourages social interaction among participants. This program has low intensity and impact levels and can be designed for all levels of participants (Aquatic Fitness, 2010). Water-based exercises such as swimming and water walking are becoming increasingly popular, as walking in an aquatic environment is an easy-to-practice way. The weight of the body due to gravity, cancels the buoyancy and the force that the water exerts on the lower extremities can effectively control and alter several ways of walking. Therefore, walking in water is adequate, not only in everyday life, but also in rehabilitation and sports injuries as mentioned previously. Several ways of walking have been proposed for body weight changes (Konishi, 1999). However, several studies indicate that research on this subject is still short. Most 
of the studies on water walking are in physiological and non-biomechanical aspects (Nakayama, 1990; Migita, Hotta, Ogaki, Kanaya, Fujishima \& Masuda, 1996). Therefore, it is important to develop a biomechanical simulation tool that can analyse the benefit that water exerts on the lower extremities to optimize the walking. Over the years, some researchers have analysed the biomechanical movements of water walking and ground/land walking, and it has been found that there are differences in cadence, range, velocity, duration of gait phases (Lopes, 2009). According to Wilder \& Brennam, (1993) and Hamer \& Slocombre, (1997), there are two phases in the water walking: The first phase is the support phase that is fragmented into initial, middle and final support. The second phase is the balance sheet phase, which is divided into three subfactors, prebalance sheet, average balance sheet and final balance sheet. Water walking has the same motor behaviour as walking in the ground/land, yet, its efficiency is completely different (Lazzari \& Meyer, 1997). Due to the viscosity of the water and knee flexion, erect posture is more difficult, this leads to increased resistance to movement (Town \& Bradley, 1991). Leite, Lazarotto, Leilane, Lopes, Bento, Torres, Heyde, Von Der, Cieslak, Milano (2010) declare that the water walking in suspension, when the intervener has a suitable weekly frequency and intensity of exercise to his level of aptitude, coupled with a nutritional program, may result in relevant changes in body composition in obese children or adolescents due to the cyclical characteristic of this activity.

\section{MATERIALS AND METHODOLOGY}

\section{Participants}

The selected participants were randomly chosen. 28 children aged between 6 and 12 years attending swimming classes. The participants were divided into two groups, the swimming group and the swimming group complemented with water walking. The swimming group were 9 children and the swimming group with water walking were 19. The swimming group did only swimming lessons while the group of swimming with water walking did swimming complemented with 6 minutes of water walking at the end of each session, as proposed and performed by Keino, Born, \& Plasqui (2014), Carvalho et al. (2015) and Lopes, Bento, Lazzaroto, Rodacki \& Leite (2015). The children who participated in the study belonged to classes of initiation and water adaptation for a group's homogeneity.

\section{Procedures}

We performed a first evaluation of body composition parameters (Weight, Muscle Mass, Fat Mass, Water Percentage), using a Targa Silvercrest Bio-Impedance Balance. For the BMI we follow the formula (weight / height2) from Cole (2000) and the abdominal perimeter assessments were based on the ISAK (International Society of the Advancement of Kinanthropometry) test, proposed by Ross and Marfell-Jones in 1991. To estimate obesity percentiles, we used the World Health Organization tables (WHO, 2007). Finally, to calculate the spirometric values we used the Cosmed Microquark spirometer to measure forced vital capacity (FVC), forced expiratory volume in 1 second (FEV1), peak expiratory flow (PEF). We carried out two further evaluations, an intermediate evaluation after six weeks and a final evaluation at the end of the twelve weeks, based on the same criteria. Swimming lessons were held in an indoor pool of $16 \mathrm{~m} \times 8 \mathrm{~m}$ size learning tank with an average depth of $0.80 \mathrm{~m}$, with a water temperature of 300 , with a weekly frequency of 2 times a week, with 45 minutes each. The classes were guided by the teachers of the institution that were responsible for these classes, using the Borg Scale to control the effort intensity, as done by Hildenbrand, Nordio, Freson, \& Becker (2010). The swim group did the whole swimming lesson as established and the swimming and water walking group spent the last 6 minutes of the session performing water walking, which is performed in a straight line near the edge of the pool, with no lateral supports (only feet support). The water was at the level of the chest according to the studies of Keino, Born, \& Plasqui (2014), Carvalho et al. (2015) and Lopes, Bento, Lazzaroto, Rodacki \& Leite (2015). 


\section{Statistical Procedures}

For statistical procedures we used the SPSS (version 20.0). Initially we presented descriptive statistics to determine the mean, standard deviation, minimum and maximum of the two groups at the different moments of the evaluations. Then, for testing the normality of the two groups it was applied the Shapiro-Wilk statistical test, since the data presented a normal distribution, we chose to perform the non-parametric tests because the participants were less than 30 . We used the non-parametric tests with two related samples and compared the distributions of each variable at each time between the control and experimental groups through the Friedman Anova test. We applied the non-parametric test between two independent samples, the MannWhitney Test, to obtain the significant differences between each group in all moments for evaluation. Statistical analysis considered the following levels of significance: $p<0.01{ }^{* *}$ very significant; $p<0.05^{*}$ significant. The method of inferences based on the magnitude of the effects was also performed. The intervals to classify the magnitude of the effects (d Cohen) were as follows: 0-0.2, trivial; 0.21-0.6, small; 0.61-1.2, moderate, 1.21-2.0, large; > 2.0, very large, according to (Hopkins, 2009).

\section{RESULTS}

We present the descriptive statistics of the two groups, as well as the results of the body composition and spirometric values, during the three moments of evaluation.

Table 1. Descriptive statistics of body composition and spirometric values in the groups of swimming and swimming water walking on the 3 moments

\begin{tabular}{|c|c|c|c|c|c|c|c|}
\hline & \multirow[b]{2}{*}{$\mathrm{N}$} & \multirow[b]{2}{*}{ Minimum } & \multirow[b]{2}{*}{ Maximum } & \multirow[b]{2}{*}{ Mean } & \multirow[b]{2}{*}{ Standard deviation } & \multicolumn{2}{|c|}{ Normality } \\
\hline & & & & & & Value & $p$ \\
\hline Height $(\mathrm{cm})$ & 19 & 1.17 & 1.49 & 1.3032 & 0.07804 & 0.799 & 0.001 \\
\hline Weight_1 $(\mathrm{kg})$ & 19 & 22.0 & 46.0 & 29.589 & 6.1511 & 0.977 & 0.898 \\
\hline Weight_2 (kg) & 19 & 22.2 & 46.1 & 30.126 & 6.0189 & 0.875 & 0.017 \\
\hline Weight_3 (kg) & 19 & 22.5 & 45.8 & 30.442 & 6.0478 & 0.903 & 0.056 \\
\hline Muscle_mass_1 $(\mathrm{kg})$ & 19 & 32.9 & 42.2 & 37.342 & 2.5338 & 0.894 & 0.037 \\
\hline Muscle_mass_2 $(\mathrm{kg})$ & 19 & 32.9 & 42.0 & 37.274 & 2.5927 & 0.981 & 0.957 \\
\hline Muscle_mass_3 (kg) & 19 & 32.8 & 42.0 & 36.737 & 2.7352 & 0.976 & 0.891 \\
\hline Fat mass_1 $1 \%$ ) & 19 & 12 & 23.7 & 17.563 & 3.567 & 0.944 & 0.309 \\
\hline Fat mass_2 (\%) & 19 & 12.5 & 23.8 & 17.474 & 3.5755 & 0.955 & 0.471 \\
\hline Fat mass_3 (\%) & 19 & 12.5 & 24.2 & 17.947 & 3.7017 & 0.941 & 0.278 \\
\hline Water_1 (\%) & 19 & 50.1 & 64.4 & 56.663 & 4.2128 & 0.940 & 0.267 \\
\hline Water_2 (\%) & 19 & 50.0 & 64.2 & 56.532 & 3.8446 & 0.971 & 0.792 \\
\hline Water_3 (\%) & 19 & 49.9 & 64.1 & 56.016 & 4.2412 & 0.975 & 0.870 \\
\hline Waist circunference_1 $(\mathrm{cm})$ & 19 & 50.5 & 71.0 & 59.474 & 5.8653 & 0.555 & 0.000 \\
\hline Waist circunference_2 $(\mathrm{cm})$ & 19 & 49.0 & 71.0 & 59.237 & 5.8199 & 0.860 & 0.010 \\
\hline Waist circunference_3 $(\mathrm{cm})$ & 19 & 52.0 & 70.0 & 59.184 & 5.3624 & 0.949 & 0.381 \\
\hline BMI_1 $\left(\mathrm{kg} / \mathrm{m}^{2}\right)$ & 19 & 14.5 & 23.3 & 17.268 & 2.3721 & 0.919 & 0.107 \\
\hline BMI_2 (kg/m²) & 19 & 14.7 & 22.8 & 17.574 & 2.2248 & 0.923 & 0.129 \\
\hline BMI_3 $\left(\mathrm{kg} / \mathrm{m}^{2}\right)$ & 19 & 14.9 & 23.3 & 17.774 & 2.3026 & 0.942 & 0.285 \\
\hline Body percentile_1(kg/m²) & 19 & 5.0 & 99.0 & 59.37 & 32.383 & 0.935 & 0.216 \\
\hline Body percentile_2 $\left(\mathrm{kg} / \mathrm{m}^{2}\right)$ & 19 & 15.0 & 99.0 & 61.47 & 31.660 & 0.877 & 0.019 \\
\hline Body percentile_3 $3\left(\mathrm{~kg} / \mathrm{m}^{2}\right)$ & 19 & 15.0 & 99.0 & 68.00 & 29.827 & 0.848 & 0.006 \\
\hline FVC_1 (I) & 19 & 0.62 & 2.44 & 1.6300 & 0.48527 & 0.807 & 0.001 \\
\hline FVC_2(I) & 19 & 0.93 & 2.51 & 1.8289 & 0.42080 & 0.975 & 0.876 \\
\hline FVC_3(I) & 19 & 0.94 & 2.60 & 1.8089 & 0.41032 & 0.967 & 0.706 \\
\hline FEV1_1 (I) & 19 & 0.58 & 2.41 & 1.5511 & 0.46523 & 0.985 & 0.986 \\
\hline FEV1_2 (I) & 19 & 0.73 & 2.48 & 1.6837 & 0.41892 & 0.970 & 0.785 \\
\hline FEV1_3 (I & 19 & 1.11 & 2.50 & 1.7084 & 0.38890 & 0.977 & 0.898 \\
\hline DEMI_1 (I) & 19 & 0.94 & 5.59 & 3.4921 & 1.16774 & 0.967 & 0.722 \\
\hline DEMI_2(I) & 19 & 1.62 & 5.55 & 3.7663 & 1.00117 & 0.933 & 0.199 \\
\hline DEMI_3(I) & 19 & 1.84 & 5.33 & 3.8705 & 1.02156 & 0.976 & 0.894 \\
\hline
\end{tabular}


Table 1 presents the data referring to the descriptive statistics of body composition in the group that performed swimming and water walking. The analysis of the table reports the values of normality in the variables of height, weight, muscle mass, fat mass, water percentage, body mass index and body percentiles.

Table 2. Inter-group body composition results in all moments

\begin{tabular}{|c|c|c|c|c|c|c|c|c|c|c|c|}
\hline & & & M1 & M2 & M3 & & M1 & & M2 & & M3 \\
\hline Variables & Groups & $\mathrm{n}$ & $M \pm s d$ & $M \pm s d$ & $M \pm s d$ & Sig. & $\begin{array}{c}\text { Differences } \\
\text { in means } \\
(\mathrm{d} ; \pm 95 \% \\
\mathrm{Cl})\end{array}$ & Sig. & $\begin{array}{c}\text { Differences } \\
\text { in means } \\
(\mathrm{d} ; \pm 95 \% \\
\mathrm{Cl})\end{array}$ & Sig. & $\begin{array}{l}\text { Differences } \\
\text { in means } \\
(\mathrm{d} ; \pm 95 \% \\
\mathrm{Cl})\end{array}$ \\
\hline weight $(\mathrm{kg})$ & $\begin{array}{l}\text { Swimming } \\
\text { and water } \\
\text { walking } \\
\text { Swimming }\end{array}$ & 19 & $23.7 \pm 3.64$ & $24.9 \pm 4.54$ & $25.5 \pm 4.38$ & $0.004^{* *}$ & $\begin{array}{c}1.07 \\
(0.34 \pm 1.75) \\
\text { moderate }\end{array}$ & $0.022^{*}$ & $\begin{array}{c}0.93 \\
(0.21 \pm 1.60) \\
\text { little }\end{array}$ & $0.037^{*}$ & $\begin{array}{c}0.88 \\
(0.16 \pm 1.54) \\
\text { little }\end{array}$ \\
\hline $\begin{array}{c}\text { Muscle } \\
\text { mass (kg) }\end{array}$ & $\begin{array}{l}\text { Swimming } \\
\text { and water } \\
\text { walking } \\
\text { Swimming }\end{array}$ & 19 & $\begin{array}{l}37.3 \pm 2.53 \\
37.3 \pm 1.44\end{array}$ & $\begin{array}{l}37.3 \pm 2.59 \\
35.2 \pm 2.89\end{array}$ & $\begin{array}{l}36.7 \pm 2.74 \\
35.9 \pm 3.56\end{array}$ & 0.923 & $\begin{array}{c}0.00(- \\
0.67 \pm 0.67) \\
\text { trivial }\end{array}$ & 0.117 & $\begin{array}{c}0.78 \\
(0.07 \pm 1.45) \\
\text { moderate }\end{array}$ & 0.629 & $\begin{array}{c}0.27(- \\
0.41 \pm 0.93) \\
\text { little }\end{array}$ \\
\hline $\begin{array}{c}\text { Fat mass } \\
(\%)\end{array}$ & $\begin{array}{l}\text { Swimming } \\
\text { and water } \\
\text { walking } \\
\text { Swimming }\end{array}$ & 19 & $\begin{array}{l}17.6 \pm 3.57 \\
18.4 \pm 2.66\end{array}$ & $\begin{array}{c}17.5 \pm 3.58 \\
19.2 \pm 4.61\end{array}$ & $\begin{array}{r}17.9 \pm 3.70 \\
19.1 \pm 4.90\end{array}$ & 0.595 & $\begin{array}{c}-0.24(- \\
0.90 \pm 0.43) \\
\quad \text { little }\end{array}$ & 0.410 & $\begin{array}{c}-0.43(- \\
1.09 \pm 0.25) \\
\text { little }\end{array}$ & 0.562 & $\begin{array}{c}-0.29(- \\
0.95 \pm 0.38) \\
\text { little }\end{array}$ \\
\hline Water (\%) & $\begin{array}{l}\text { Swimming } \\
\text { and water } \\
\text { walking } \\
\text { Swimming }\end{array}$ & 19 & $53.8 \pm 2.10$ & $53.6 \pm 4.44$ & $\begin{array}{l}56.0 \pm 4.24 \\
53.5 \pm 4.42\end{array}$ & 0.095 & $\begin{array}{c}0.79 \\
(0.08 \pm 1.45) \\
\text { moderate }\end{array}$ & 0.129 & $\begin{array}{c}0.72 \\
(0.01 \pm 1.38) \\
\text { moderate }\end{array}$ & 0.308 & $\begin{array}{c}0.58(- \\
0.11 \pm 1.24) \\
\text { little }\end{array}$ \\
\hline $\begin{array}{c}\text { Waist } \\
\text { perimeter } \\
(\mathrm{cm})\end{array}$ & $\begin{array}{l}\text { Swimming } \\
\text { and water } \\
\text { walking } \\
\text { Swimming }\end{array}$ & 19 & $\begin{array}{l}59.5 \pm 5.87 \\
54.4 \pm 5.17\end{array}$ & $\begin{array}{l}59.2 \pm 5.82 \\
56.3 \pm 5.73\end{array}$ & $\begin{array}{l}59.2 \pm 5.36 \\
56.0 \pm 5.38\end{array}$ & $0.007^{* *}$ & $\begin{array}{c}0.90 \\
(0.18 \pm 1.57) \\
\text { moderate }\end{array}$ & 0.085 & $\begin{array}{c}0.50(- \\
0.19 \pm 1.16) \\
\quad \text { little }\end{array}$ & 0.117 & $\begin{array}{c}0.60(- \\
0.10 \pm 1.26) \\
\text { little }\end{array}$ \\
\hline $\begin{array}{c}\text { BMl } \\
\left(\mathrm{kg} / \mathrm{m}^{2}\right)\end{array}$ & $\begin{array}{l}\text { Swimming } \\
\text { and water } \\
\text { walking } \\
\text { Swimming }\end{array}$ & 19 & $\begin{array}{l}17.3 \pm 2.37 \\
15.4 \pm 1.72\end{array}$ & $17.6 \pm 2.22$ & $\begin{array}{l}17.8 \pm 2.30 \\
16.7 \pm 2.70\end{array}$ & $0.028^{*}$ & $\begin{array}{c}0.87 \\
(0.15 \pm 1.53) \\
\text { moderate }\end{array}$ & 0.068 & $\begin{array}{c}0.54(- \\
0.15 \pm 1.20) \\
\quad \text { little }\end{array}$ & 0.142 & $\begin{array}{c}0.45(- \\
0.23 \pm 1.11) \\
\text { little }\end{array}$ \\
\hline $\begin{array}{c}\text { Body } \\
\text { percentiles } \\
\left(\mathrm{kg} / \mathrm{m}^{2}\right)\end{array}$ & $\begin{array}{l}\text { Swimming } \\
\text { and water } \\
\text { walking } \\
\text { Swimming }\end{array}$ & 19 & $\begin{array}{l}59.4 \pm 32.4 \\
35.0 \pm 31.1\end{array}$ & $\begin{array}{l}61.5 \pm 31.7 \\
41.0 \pm 32.4\end{array}$ & $\begin{array}{l}68.0 \pm 29.8 \\
46.0 \pm 30.0\end{array}$ & 0.054 & $\begin{array}{c}0.76(- \\
0.05 \pm 1.27) \\
\text { moderate }\end{array}$ & 0.129 & $\begin{array}{c}0.64(- \\
0.06 \pm 1.30) \\
\text { moderate }\end{array}$ & 0.117 & $\begin{array}{c}0.74 \\
(0.03 \pm 1.40) \\
\text { moderate }\end{array}$ \\
\hline
\end{tabular}

M1: Moment 1; M2: Moment 2; M3: Moment

Table 2 refers to the spirometric values in the group that did the swimming lessons supplemented with water walking. The values that are referenced are forced vital capacity, forced expiratory volume in $1 \mathrm{sec}$. and peak expiratory flow.

Table 3. Inter-group spirometric results in all moments

\begin{tabular}{|c|c|c|c|c|c|c|c|c|c|c|c|}
\hline & & & M1 & M2 & M3 & \multicolumn{2}{|r|}{ M1 } & \multicolumn{2}{|r|}{ M2 } & \multicolumn{2}{|r|}{ M3 } \\
\hline Variables & Groups & $\mathrm{n}$ & $M \pm s d$ & $M \pm s d$ & $M \pm s d$ & Sig. & $\begin{array}{c}\text { Differences } \\
\text { in means } \\
\text { (d; } \pm 95 \% \\
\mathrm{Cl})\end{array}$ & Sig. & $\begin{array}{c}\text { Differences } \\
\text { in means (d; } \\
\pm 95 \% \mathrm{Cl} \text { ) }\end{array}$ & Sig. & $\begin{array}{c}\text { Differences } \\
\text { in means (d; } \\
\pm 95 \% \mathrm{Cl} \text { ) }\end{array}$ \\
\hline FVC (I) & $\begin{array}{c}\text { Swimming } \\
\text { and water } \\
\text { walking }\end{array}$ & 19 & $1.63 \pm 0.49$ & $1.83 \pm 0.42$ & $1.81 \pm 0.41$ & 0.285 & $\begin{array}{c}0.33(- \\
0.35 \pm 0.99) \\
\text { little }\end{array}$ & $0.025^{*}$ & $\begin{array}{c}0.92 \\
(0.20 \pm 1.59) \\
\text { moderate }\end{array}$ & 0.054 & $\begin{array}{c}0.76 \\
(0.06 \pm 1.43) \\
\text { moderate }\end{array}$ \\
\hline & Swimming & 9 & $1.48 \pm 0.34$ & $1.47 \pm 0.31$ & $1.52 \pm 0.30$ & & & & & & \\
\hline VEF (I) & $\begin{array}{c}\text { Swimming } \\
\text { and water } \\
\text { walking }\end{array}$ & 19 & $1.55 \pm 0.47$ & $1.68 \pm 0.42$ & $1.71 \pm 0.39$ & 0.068 & $\begin{array}{c}0.66(- \\
0.04 \pm 1.32) \\
\text { moderate }\end{array}$ & $0.010^{*}$ & $\begin{array}{c}0.94 \\
(0.22 \pm 1.61) \\
\text { moderate }\end{array}$ & $0.028^{*}$ & $\begin{array}{c}0.84 \\
(0.13 \pm 1.51) \\
\text { moderate }\end{array}$ \\
\hline & Swimming & 9 & $1.28 \pm 0.21$ & $1.33 \pm 0.23$ & $1.41 \pm 0.26$ & & & & & & \\
\hline PEF (I) & $\begin{array}{c}\text { Swimming } \\
\text { and water } \\
\text { walking }\end{array}$ & 19 & $3.49 \pm 1.17$ & $3.77 \pm 1.00$ & $3.87 \pm 1.02$ & $0.033^{*}$ & $\begin{array}{c}0.63(- \\
0.06 \pm 1.29) \\
\text { moderate }\end{array}$ & $0.012^{*}$ & $\begin{array}{c}1.03 \\
(0.30 \pm 1.70) \\
\text { moderate }\end{array}$ & $0.037^{*}$ & $\begin{array}{c}0.77 \\
(0.06 \pm 1.43) \\
\text { moderate }\end{array}$ \\
\hline & Swimming & 9 & $2.85 \pm 0.49$ & $2.88 \pm 0.43$ & $3.19 \pm 0.44$ & & & & & & \\
\hline
\end{tabular}

M1: Moment 1; M2: Moment 2; M3: Moment; FVC: Forced vital capacity; FEV 1: forced expiratory volume in 1 sec; PEF: peak expiratory flow. 
Table 3 represents the comparison of the moments between the swimming group and the swimming group complemented with water walking, in relation to body composition.

Table 4. Intra-group results of body composition

\begin{tabular}{|c|c|c|c|c|c|c|c|c|c|c|c|c|}
\hline & & & M1 & M2 & M3 & & 11-M2 & & 12-M3 & & 1-M3 & $\begin{array}{c}\text { M1- } \\
\text { M2-M3 }\end{array}$ \\
\hline Variables & Groups & $n$ & $M \pm s d$ & $M \pm s d$ & $M \pm s d$ & Sig. & $\begin{array}{c}\text { Difference } \\
\text { s in means } \\
(\mathrm{d} ; \pm 95 \% \\
\mathrm{Cl})\end{array}$ & Sig. & $\begin{array}{c}\text { Difference } \\
\text { s in means } \\
(\mathrm{d} ; \pm 95 \% \\
\mathrm{Cl})\end{array}$ & Sig. & $\begin{array}{c}\text { Difference } \\
\text { s in means } \\
\text { (d; } \pm 95 \% \\
\text { Cl) }\end{array}$ & Sig. \\
\hline \multirow{2}{*}{$\begin{array}{l}\text { Weight } \\
(\mathrm{kg})\end{array}$} & $\begin{array}{c}\text { Swimmin } \\
g \text { and } \\
\text { water } \\
\text { walking }\end{array}$ & $\begin{array}{l}1 \\
9\end{array}$ & $\begin{array}{c}29.6 \pm 6.1 \\
4\end{array}$ & $\begin{array}{c}30.1 \pm 6.0 \\
1\end{array}$ & $\begin{array}{c}30.4 \pm 6.0 \\
5\end{array}$ & $\underset{*}{0.004^{*}}$ & $\begin{array}{c}-0.08(- \\
0.61 \pm 0.45) \\
\text { trivial }\end{array}$ & $\begin{array}{c}0.58 \\
3\end{array}$ & $\begin{array}{c}-0.05(- \\
0.58 \pm 0.49 \\
\text { ) trivial }\end{array}$ & $\underset{*}{0.000^{*}}$ & $\begin{array}{c}-0.13(- \\
0.66 \pm 0.41 \\
\text { ) trivial }\end{array}$ & $\underset{*}{0.000^{*}}$ \\
\hline & $\begin{array}{c}\text { Swimmin } \\
\mathrm{g}\end{array}$ & 9 & $\begin{array}{c}23.7 \pm 3.6 \\
4\end{array}$ & $\begin{array}{c}24.9 \pm 4.5 \\
4\end{array}$ & $\begin{array}{c}25.5 \pm 4.3 \\
8\end{array}$ & 0.297 & $\begin{array}{c}-0.29(- \\
1.06 \pm 0.50) \\
\quad \text { little }\end{array}$ & $\begin{array}{c}0.29 \\
7\end{array}$ & $\begin{array}{c}-0.13(- \\
0.90 \pm 0.65 \\
\text { ) trivial }\end{array}$ & $\underset{*}{0.003^{*}}$ & $\begin{array}{c}-0.45(- \\
1.21 \pm 0.36 \\
) \text { little }\end{array}$ & $\underset{*}{0.004^{*}}$ \\
\hline \multirow{2}{*}{$\begin{array}{c}\text { Muscle } \\
\text { mass (kg) }\end{array}$} & $\begin{array}{c}\text { Swimmin } \\
\mathrm{g} \text { and } \\
\text { water } \\
\text { walking }\end{array}$ & $\begin{array}{l}1 \\
9\end{array}$ & $\begin{array}{c}37.3 \pm 2.5 \\
3\end{array}$ & $\begin{array}{c}37.3 \pm 2.5 \\
9\end{array}$ & $\begin{array}{c}36.7 \pm 2.7 \\
4\end{array}$ & \multirow[t]{2}{*}{1.000} & $\begin{array}{c}0.00(- \\
0.67 \pm 0.67) \\
\quad \text { trivial }\end{array}$ & $\begin{array}{c}0.18 \\
6\end{array}$ & $\begin{array}{c}0.23(- \\
0.31 \pm 0.76 \\
\text { ) little }\end{array}$ & \multirow[t]{2}{*}{0.069} & $\begin{array}{c}0.23(- \\
0.31 \pm 0.76 \\
\text { ) little }\end{array}$ & $0.029^{*}$ \\
\hline & $\begin{array}{c}\text { Swimmin } \\
\mathrm{g}\end{array}$ & 9 & $\begin{array}{c}37.3 \pm 1.4 \\
4\end{array}$ & $\begin{array}{c}35.2 \pm 2.8 \\
9\end{array}$ & $\begin{array}{c}35.9 \pm 3.5 \\
6\end{array}$ & & $\begin{array}{c}0.92 \\
(0.06 \pm 1.69 \\
\text { ) moderate }\end{array}$ & & $\begin{array}{c}-0.22(- \\
0.98 \pm 0.57 \\
) \text { little }\end{array}$ & & $\begin{array}{c}0.52(- \\
0.30 \pm 1.28 \\
) \text { little }\end{array}$ & 0.075 \\
\hline \multirow{2}{*}{$\begin{array}{c}\text { Fat mass } \\
(\%)\end{array}$} & $\begin{array}{c}\text { Swimmin } \\
\mathrm{g} \text { and } \\
\text { water } \\
\text { walking }\end{array}$ & $\begin{array}{l}1 \\
9\end{array}$ & $\begin{array}{c}17.6 \pm 3.5 \\
7\end{array}$ & $\begin{array}{c}17.5 \pm 3.5 \\
8\end{array}$ & $\begin{array}{c}17.9 \pm 3.7 \\
0\end{array}$ & \multirow[t]{2}{*}{0.583} & $\begin{array}{c}0.03(- \\
0.51 \pm 0.56) \\
\text { trivial }\end{array}$ & $\begin{array}{c}0.12 \\
8\end{array}$ & $\begin{array}{c}-0.11(- \\
0.64 \pm 0.43 \\
\text { ) trivial }\end{array}$ & \multirow[t]{2}{*}{$\underset{*}{0.003^{*}}$} & $\begin{array}{c}-0.08(- \\
0.61 \pm 0.45 \\
\text { ) trivial }\end{array}$ & $\underset{*}{0.002^{*}}$ \\
\hline & $\begin{array}{c}\text { Swimmin } \\
\mathrm{g}\end{array}$ & 9 & $\begin{array}{c}18.4 \pm 2.6 \\
6\end{array}$ & $\begin{array}{c}19.2 \pm 4.6 \\
1\end{array}$ & $\begin{array}{c}19.1 \pm 4.9 \\
0\end{array}$ & & $\begin{array}{c}-0.21(- \\
0.98 \pm 0.57) \\
\text { little }\end{array}$ & & $\begin{array}{c}0.02(- \\
0.76 \pm 0.80 \\
\text { ) trivial }\end{array}$ & & $\begin{array}{c}-0.18(- \\
0.95 \pm 0.61 \\
\text { ) trivial }\end{array}$ & 0.196 \\
\hline \multirow[t]{2}{*}{ Water (\%) } & $\begin{array}{c}\text { Swimmin } \\
\text { g and } \\
\text { water } \\
\text { walking }\end{array}$ & $\begin{array}{l}1 \\
9\end{array}$ & $\begin{array}{c}56.7 \pm 4.2 \\
1\end{array}$ & $\begin{array}{c}56.5 \pm 3.8 \\
4\end{array}$ & $\begin{array}{c}56.0 \pm 4.2 \\
4\end{array}$ & \multirow[t]{2}{*}{1.000} & $\begin{array}{c}0.05(- \\
0.49 \pm 0.58) \\
\text { trivial }\end{array}$ & $\begin{array}{l}0.10 \\
5\end{array}$ & $\begin{array}{c}0.12(- \\
0.41 \pm 0.66 \\
\text { ) trivial }\end{array}$ & \multirow[t]{2}{*}{$0.036^{*}$} & $\begin{array}{c}0.17(- \\
0.37 \pm 0.70 \\
\text { ) trivial }\end{array}$ & $0.018^{*}$ \\
\hline & $\begin{array}{c}\text { Swimmin } \\
\mathrm{g}\end{array}$ & 9 & $\begin{array}{c}53.8 \pm 2.1 \\
0\end{array}$ & $\begin{array}{c}53.6 \pm 4.4 \\
4\end{array}$ & $\begin{array}{c}53.5 \pm 4.4 \\
2\end{array}$ & & $\begin{array}{c}0.06(- \\
0.72 \pm 0.83) \\
\text { trivial }\end{array}$ & & $\begin{array}{c}0.02(- \\
0.75 \pm 0.80 \\
\text { ) trivial }\end{array}$ & & $\begin{array}{c}0.09(- \\
0.69 \pm 0.86 \\
\text { ) trivial }\end{array}$ & 0.157 \\
\hline \multirow{2}{*}{$\begin{array}{c}\text { Waist } \\
\text { perimeter } \\
(\mathrm{cm})\end{array}$} & $\begin{array}{c}\text { Swimmin } \\
\text { g and } \\
\text { water } \\
\text { walking }\end{array}$ & $\begin{array}{l}1 \\
9\end{array}$ & $\begin{array}{c}59.5 \pm 5.8 \\
7\end{array}$ & $\begin{array}{c}59.2 \pm 5.8 \\
2\end{array}$ & $\begin{array}{c}59.2 \pm 5.3 \\
6\end{array}$ & & $\begin{array}{c}0.05(- \\
0.48 \pm 0.58) \\
\quad \text { trivial }\end{array}$ & & $\begin{array}{c}0.00(- \\
0.53 \pm 0.53 \\
\text { ) trivial }\end{array}$ & & $\begin{array}{c}0.05(- \\
0.48 \pm 0.59 \\
\text { ) trivial }\end{array}$ & 0.550 \\
\hline & $\begin{array}{c}\text { Swimmin } \\
\mathrm{g}\end{array}$ & 9 & $\begin{array}{c}54.4 \pm 5.1 \\
7\end{array}$ & $\begin{array}{c}56.3 \pm 5.7 \\
3\end{array}$ & $\begin{array}{c}56.0 \pm 5.3 \\
8\end{array}$ & $\underset{*}{0.004^{*}}$ & $\begin{array}{c}-0.35(- \\
1.11 \pm 0.45) \\
\text { little }\end{array}$ & $\begin{array}{c}0.86 \\
7\end{array}$ & $\begin{array}{c}-0.05(- \\
0.72 \pm 0.83 \\
\text { ) trivial }\end{array}$ & 0.102 & $\begin{array}{c}-0.30(- \\
1.07 \pm 0.49 \\
\text { ) trivial }\end{array}$ & $\underset{*}{0.005^{*}}$ \\
\hline \multirow{2}{*}{$\begin{array}{c}\text { BMl } \\
\left(\mathrm{kg} / \mathrm{m}^{2}\right)\end{array}$} & $\begin{array}{c}\text { Swimmin } \\
\text { g and } \\
\text { water } \\
\text { walking }\end{array}$ & $\begin{array}{l}1 \\
9\end{array}$ & $\begin{array}{c}17.3 \pm 2.3 \\
7\end{array}$ & $\begin{array}{c}17.6 \pm 2.2 \\
2\end{array}$ & $\begin{array}{c}17.8 \pm 2.3 \\
0\end{array}$ & $\underset{*}{0.008^{*}}$ & $\begin{array}{c}-0.08(- \\
0.61 \pm 0.45) \\
\text { trivial }\end{array}$ & $\begin{array}{c}0.58 \\
3\end{array}$ & $\begin{array}{c}-0.05(- \\
0.58 \pm 0.49 \\
\text { ) trivial }\end{array}$ & $\underset{*}{0.000^{*}}$ & $\begin{array}{c}-0.13(- \\
0.66 \pm 0.41 \\
\text { ) trivial }\end{array}$ & $\underset{*}{0.000^{*}}$ \\
\hline & $\begin{array}{c}\text { Swimmin } \\
\mathrm{g}\end{array}$ & 9 & $\begin{array}{c}15.4 \pm 1.7 \\
2\end{array}$ & $\begin{array}{c}16.3 \pm 2.7 \\
6\end{array}$ & $\begin{array}{c}16.7 \pm 2.7 \\
0\end{array}$ & 0.231 & $\begin{array}{c}-0.29(- \\
1.06 \pm 0.50) \\
\text { little }\end{array}$ & $\begin{array}{c}0.47 \\
2\end{array}$ & $\begin{array}{c}-0.13(- \\
0.90 \pm 0.65 \\
\text { ) trivial }\end{array}$ & $\underset{*}{0.004^{*}}$ & $\begin{array}{c}-0.45(- \\
1.21 \pm 0.36 \\
) \text { little }\end{array}$ & $\underset{*}{0.005^{*}}$ \\
\hline \multirow{2}{*}{$\begin{array}{c}\text { Body } \\
\text { percentile } \\
\mathrm{s}\left(\mathrm{kg} / \mathrm{m}^{2}\right)\end{array}$} & $\begin{array}{c}\text { Swimmin } \\
g \text { and } \\
\text { water } \\
\text { walking }\end{array}$ & $\begin{array}{l}1 \\
9\end{array}$ & $\begin{array}{c}59.4 \pm 32 \\
4\end{array}$ & $\begin{array}{c}61.5 \pm 31 \\
7\end{array}$ & $\begin{array}{c}68.0 \pm 29 \\
8\end{array}$ & 1.000 & $\begin{array}{c}-0.07(- \\
0.60 \pm 0.47) \\
\text { trivial }\end{array}$ & $\begin{array}{c}0.18 \\
6\end{array}$ & $\begin{array}{c}-0.21(- \\
0.74 \pm 0.33 \\
\text { ) little }\end{array}$ & $0.017^{*}$ & $\begin{array}{c}-0.42(- \\
0.95 \pm 0.13 \\
\text { ) little }\end{array}$ & $\underset{*}{0.000^{*}}$ \\
\hline & $\begin{array}{c}\text { Swimmin } \\
\mathrm{g}\end{array}$ & 9 & $\begin{array}{c}35.0 \pm 31 \\
1\end{array}$ & $\begin{array}{c}41.0 \pm 32 \\
4\end{array}$ & $\begin{array}{c}46.0 \pm 30 \\
0\end{array}$ & 0.716 & $\begin{array}{c}-0.19(- \\
0.96 \pm 0.60) \\
\text { trivial }\end{array}$ & $\begin{array}{c}1.00 \\
0\end{array}$ & $\begin{array}{c}-0.16(- \\
0.93 \pm 0.62 \\
\text { ) trivial }\end{array}$ & 0.135 & $\begin{array}{c}-0.36(- \\
1.12 \pm 0.44 \\
\text { ) little }\end{array}$ & $\underset{*}{0.014^{*}}$ \\
\hline
\end{tabular}

Table 4 represents the comparison of the moments between the swimming group and swimming group with water walking in relation to the spirometric values. 
Table 5. Intra-group results of spirometric values

\begin{tabular}{|c|c|c|c|c|c|c|c|c|c|c|c|c|}
\hline \multirow[b]{2}{*}{$\begin{array}{c}\text { Variable } \\
\mathrm{s}\end{array}$} & \multirow[b]{2}{*}{ Groups } & \multirow[b]{2}{*}{$\mathrm{n}$} & \multirow{2}{*}{$\begin{array}{c}\mathrm{M} 1 \\
\mathrm{M} \pm \mathrm{sd}\end{array}$} & \multirow{2}{*}{$\begin{array}{c}\mathrm{M} 2 \\
\mathrm{M} \pm \mathrm{sd}\end{array}$} & \multirow{2}{*}{$\begin{array}{c}\text { M3 } \\
M \pm s d\end{array}$} & \multicolumn{2}{|c|}{ M1-M2 } & \multicolumn{2}{|c|}{ M2-M3 } & \multicolumn{2}{|c|}{ M1-M3 } & \multirow{2}{*}{$\begin{array}{c}\text { M1- } \\
\text { M2-M3 } \\
\text { Sig. }\end{array}$} \\
\hline & & & & & & Sig. & $\begin{array}{c}\text { Difference } \\
\text { s in means } \\
(\mathrm{d} ; \pm 95 \% \\
\mathrm{Cl})\end{array}$ & Sig. & $\begin{array}{c}\text { Difference } \\
\text { s in means } \\
(\mathrm{d} ; \pm 95 \% \\
\mathrm{Cl})\end{array}$ & Sig. & $\begin{array}{c}\text { Difference } \\
\text { s in means } \\
(\mathrm{d} ; \pm 95 \% \\
\mathrm{Cl})\end{array}$ & \\
\hline \multirow{2}{*}{ CVF (I) } & $\begin{array}{l}\text { Swimmin } \\
\text { g and } \\
\text { water } \\
\text { walking }\end{array}$ & $\begin{array}{l}1 \\
9\end{array}$ & $\begin{array}{c}1.63 \pm 0.4 \\
9\end{array}$ & $\begin{array}{c}1.83 \pm 0.4 \\
2\end{array}$ & $\begin{array}{c}1.81 \pm 0.4 \\
1\end{array}$ & $\underset{*}{0,008^{*}}$ & $\begin{array}{c}-0.44(- \\
0.97 \pm 0.11) \\
\quad \text { little }\end{array}$ & 1,000 & $\begin{array}{c}-0,05(- \\
0.49 \pm 0.58) \\
\text { trivial }\end{array}$ & $\underset{*}{0,014}$ & $\begin{array}{c}-0,40(- \\
0.93 \pm 0.15) \\
\text { little }\end{array}$ & $\underset{*}{0,003^{*}}$ \\
\hline & $\begin{array}{c}\text { Swimmin } \\
\mathrm{g}\end{array}$ & 9 & $\begin{array}{c}1.48 \pm 0.3 \\
4\end{array}$ & $\begin{array}{c}1.47 \pm 0.3 \\
1\end{array}$ & $\begin{array}{c}1.52 \pm 0.3 \\
0\end{array}$ & & $\begin{array}{c}0.03(- \\
0.75 \pm 0,80) \\
\text { trivial }\end{array}$ & & $\begin{array}{c}-0.16(- \\
0.93 \pm 0.62) \\
\text { trivial }\end{array}$ & & $\begin{array}{c}-0.12(- \\
0.89 \pm 0.66) \\
\text { trivial }\end{array}$ & 0,105 \\
\hline \multirow{2}{*}{ VEF1(I) } & $\begin{array}{l}\text { Swimmin } \\
g \text { and } \\
\text { water } \\
\text { walking }\end{array}$ & $\begin{array}{l}1 \\
9\end{array}$ & $\begin{array}{c}1.55 \pm 0.4 \\
7\end{array}$ & $\begin{array}{c}1.68 \pm 0.4 \\
2\end{array}$ & $\begin{array}{c}1.71 \pm 0.3 \\
9\end{array}$ & $0,028^{*}$ & $\begin{array}{c}-0.29(- \\
0.82 \pm 0.25) \\
\text { little }\end{array}$ & 1,000 & $\begin{array}{c}-0,07(- \\
0.61 \pm 0.46) \\
\quad \text { trivial }\end{array}$ & $\underset{*}{0,017}$ & $\begin{array}{c}-0.37(- \\
0.90 \pm 0.18) \\
\text { little }\end{array}$ & $\underset{*}{0,008^{*}}$ \\
\hline & $\begin{array}{c}\text { Swimmin } \\
\mathrm{g}\end{array}$ & 9 & $\begin{array}{c}1.28 \pm 0.2 \\
1\end{array}$ & $\begin{array}{c}1.33 \pm 0.2 \\
3\end{array}$ & $\begin{array}{c}1.41 \pm 0.2 \\
6\end{array}$ & 1,000 & $\begin{array}{c}-0.23(- \\
0.99 \pm 0.56) \\
\quad \text { little }\end{array}$ & 0,102 & $\begin{array}{c}-0.33(- \\
1.09 \pm 0.47) \\
\text { little }\end{array}$ & 0,102 & $\begin{array}{c}-0.55(- \\
1.31 \pm 0.26) \\
\text { little }\end{array}$ & $0,034^{*}$ \\
\hline \multirow{2}{*}{ PFE (I) } & $\begin{array}{l}\text { Swimmin } \\
\text { g and } \\
\text { water } \\
\text { walking }\end{array}$ & $\begin{array}{l}1 \\
9\end{array}$ & $\begin{array}{c}3.50 \pm 1.1 \\
7\end{array}$ & $\begin{array}{c}3.77 \pm 1.0 \\
0\end{array}$ & $\begin{array}{c}3.87 \pm 1.0 \\
2\end{array}$ & & $\begin{array}{l}-0.25(- \\
0.78 \pm 0.29) \\
\text { little }\end{array}$ & & $\begin{array}{c}-0,10(- \\
0.63 \pm 0.44) \\
\text { trivial }\end{array}$ & & $\begin{array}{c}-0.34(- \\
0.87 \pm 0.21) \\
\text { little }\end{array}$ & 0,060 \\
\hline & $\begin{array}{c}\text { Swimmin } \\
\mathrm{g}\end{array}$ & 9 & $\begin{array}{c}2.85 \pm 0.4 \\
9\end{array}$ & $\begin{array}{c}2.88 \pm 0.4 \\
3\end{array}$ & $\begin{array}{c}3.19 \pm 0.4 \\
4\end{array}$ & 1,000 & $\begin{array}{c}-0.07(- \\
0.84 \pm 0,71) \\
\text { trivial }\end{array}$ & $\underset{*}{0,014}$ & $\begin{array}{c}-0.71(- \\
1.48 \pm 0.12) \\
\text { moderate }\end{array}$ & 0,102 & $\begin{array}{c}-0.73(- \\
1.49 \pm 0.10) \\
\text { moderate }\end{array}$ & $0,013^{*}$ \\
\hline
\end{tabular}

M1: Moment 1; M2: Moment 2; M3: Moment; FVC: Forced vital capacity; FEV 1: forced expiratory volume in 1 sec; PEF: peak expiratory flow

Table 5 represents the comparison of the moments between the swimming group and swimming group with water walking in relation to the spirometric values.

\section{DISCUSSION}

Our study reveals significant improvements in the variables of weight, fat mass, muscle mass, water percentage, BMI and body percentiles in the inter-group and intra-group evaluations. Regarding to the study of Lopes, Bento, Lazzaroto, Rodacki, \& Leite (2015) with 73 individuals in which one group practiced water walking with nutritional orientation and the other group practiced only swimming, it was verified that in terms of body composition, BMI values, abdominal perimeter and weight did not present significant differences. The results of our investigation are not according with this study since in these same variables we present significant differences in the group that practice swimming with water walking. Another study that we highlight is the one of Bianchi, Augusto, \& Keller (2013) where they intend to evaluate the behaviour of respiratory muscle strength, peak expiratory flow and blood oxygenation during rest and walking in water. The sample consisted of 20 healthy men aged between 30 and 50 years from the respiratory point of view, where they were submitted to 4 distinct evaluations, one at rest immersed in the depth at the chest level, and another 3 , walking for 20 minutes each one of them in different depths: waist level, chest and shoulder level. At rest, there was a significant reduction of peak flow and inspiratory muscle strength during immersion, and it was concluded that peak expiratory flow (PEF) and respiratory muscle strength decreased at all levels of immersion. In this sense our study is not in agreement with these results obtained since we report statistically significant differences in the evaluation between groups in the peak of expiratory flow (PEF), and in the SWWG group, although they were not significant, there were improvements from the first to the last evaluation where the water walking was performed by the participants at the level of the chest. A study investigated by Konstantinos, Apostolos, Savvas (2007) which consisted of analysing the effects of resistance training combined with aerobic training on land and water in patients with coronary disease. The 
sample consisted of 34 individuals whose exercise took place with a weekly frequency of 4 training, 2 of resistance and another 2 of aerobic activities for each environment, that is, in land and in water, for 4 months. After 4 months of practice, it was observed that the weight variable presented significant differences in both exercise groups (exercise group on land and exercise group in water). However, in relation to the difference between the exercise groups, there was a greater difference in the group that exercised in the water in the weight variable. This result is in accordance with our study, since we had significant differences in the weight variable, both in the swimming group with water walking and in the group that only practiced swimming. The objective of this study was to propose the creation of a protocol for aquatic exercises (including immersed walking) for the development of cardiorespiratory, muscular, physical and flexibility resistance during a period of 12 weeks for individuals diagnosed with asthma. The sample consisted of 16 asthmatic individuals (9 women and 7 men). To measure the intensity of exercise, the Borg scale was used, and at the level of spirometry evaluation the parameters of FVC and FEV1 were analysed where there were no changes with the aquatic exercise protocol. Our study agrees with the use of the Borg scale in intensity control, however it is not in agreement with our values of FVC and FEV1, since our study showed statistical differences in these variables.

\section{CONCLUSIONS}

After comparing with other studies, we conclude that water walking may be an extremely interesting activity for an improvement of body composition and spirometric values and that future studies can be elaborated from this. We observed in our study that there were differences regarding weight, forced expiratory volume in 1 second and peak expiratory flow, however and according to some of these investigations if we applied more time of practical study and with a larger sample we could have better results both at the level of body composition as well as at the level of spirometric values. In a general analysis of this research, we verified the relevance of these two activities in improving parameters both at the level of body composition and at the level of spirometric values, and that they are promoters for child health, as reported by Martins (2006). The swimming program complemented with a 12-week water walk presented significant differences in relation to the variables weight, FEV1 and PEF between the SG and the SWWG group, and significant improvements in the variables of weight, abdominal circumference, body percentiles, body mass index, FVC, FEV1 in children of the SWWG group. We understand that water walking can be an attractive activity in the context of aquatic activities, which is easy to perform and shows positive effects that can help prevent childhood obesity and promote physical fitness in children in this age group.

\section{REFERENCES}

Aquatic Exercise Association. (2010). Aquatic Fitness - Profissional Manual (6th ed.) USA: Human Kinetics.

Bianchi, P. D., Augusto, I. K., \& Keller, K. D. (2013). Comportamento da força muscular respiratória, pico de fluxo expiratório e oxigenação durante repouso e caminhada na água. Fisioterapia Brasil, 14, 441-444.

Carvalho, D., Braga, E., Lochini, A., Probst, V., Pitta, F., \& Felcar, J. (2015). Functional exercise capacity evaluation in an aquatic environment. Fisioterapia e Pesquisa, 22, 359-361.

Chu, K., \& Rhodes, E. (2001). Physiological and cardiovascular chanfe associated with deep water running in the young: Possible implications for the elderly. Journal of Sports Medicine, 31, 33-46. https://doi.org/10.2165/00007256-200131010-00003

Cole, T. E. (2000). Establishing a standard definition for child overweight and obesity worldwide: international survey. British Medical Jounal, 13, 1-6. https://doi.org/10.1136/bmj.320.7244.1240 
Dowzer, C., \& Reilly, T. (1998). Deep Water Running . Ergonomics Journal, 42, 275-81. https://doi.org/10.1080/001401399185649

Faigenbaum, A. (2015). Physical Activity in Children and Adolescents. USA: American College of Sports Medicine.

Hamer, P., Slocombe, B. (1997). The Psychology and heart rate relationship between treadmill and deepwater running. Australian Physiotherapy, 43, 265-271. https://doi.org/10.1016/S00049514(14)60415-3

Hildenbrand, K., Nordio, S., Freson,T. \& Becker, B. (2010). Development of an Aquatic Exercise Training Protocol for the Asthmatic Population. International Journal of Aquatic Research and Education, 4, 278-299. https://doi.org/10.25035/ijare.04.03.07

Katzmarzyk, P., Dentro, K., Beals, K., Crouter, S., Eisenmann, J., McKenzie, T., Metz, D. (2014). Physical Activity Guidelines for Americans. USA: United States Government Printing Office.

Keino, S., Born, B. v., \& Plasqui, G. (2014). Body composition water turnover and physical activity among women in Narok County, Kenya . BMC Public Health, Vol. 14, pp: 5-8. London, England. https://doi.org/10.1186/1471-2458-14-1212

Konishi, K. (1999). Walking in water. Netherlands: Liber Press.

Konstantinos A., Apostolos Th. \& Savvas P. (2007). Land versus water exercise in patients with coronary artery disease: effects on body composition, blood lipids, and physical fitness. American Heart Journal, 154, 560-566.

Kristensen, P., Moeller, N., Korsholm, L., Kolle, E., Wedderkopp, N., Froberg, K. \& Andersen, L. (2010). The association between aerobic fitness and physical activity in children and adolescents: European Journal of Applied Physiology, 110, 267-75. https://doi.org/10.1007/s00421-010-1491-x

Kruel, L. (2000). Alterações fisiologica e biomecânicas em individuos praticando exercicios de hidroginástoca dentro e fora de água. Dissertação de Doutoramento, Universidade Federal de Santa Catarina, Brasil.

Lazzari, J., \& Meyer, F. (1997). Frequência Cardíaca e Percepção de Esforço na Caminhada Aquática e na Esteira em mulheres sedentárias e com diferentes percentuais de gordura. Revista Brasileira de Atividade Física e Saúde, 2, 07-13.

Leite, N., Lazarotto, L., Cavazza, J. F., Lopes, M. d., Bento, P. C., Torres, R., \& Milano, G. E. (2010). Efeitos de exercícios aquáticos e orientação nutricional na composição corporal de crianças e adolescentes obesos. Revista Brasileira de Cineantropometria e Desempenho Humano, 12, 235237.

Lopes, M. (2009). Caminhada aquática em suspensão e orientação nutricional em crianças e adolescentes obesos. Dissertação de Mestrado, Universidade de Coritiba, Brasil.

Lopes, M. d., Bento, P. C., Lazzaroto, L., Rodacki, A. F., \& Leite, N. (2015). The effects of water walking on the anthropometrics and metabolic aspects in young obese. Revista Brasileira de Cineantropometria \& Desempenho Humano, 17, 235-237.

Migita, T., Hotta, N., Ogaki, T., Kanaya, S., Fujishima, K., \& Masuda, T. (1996). Comparison of the physiolosical responses to treadmill prolanged walking in water and on land. Japan Journal of Physical Education, Health and Sport Sciences, 40, 316-323. https://doi.org/10.5432/ijpehss.KJ00003391415

McGlone, S., Venn, A., Walters, E., \& Wood-Baker, R. (2006). Physical activity, spirometry and qualityof-life in chronic obstructive pulmonary disease. Respiratory Care, 54, 1050-57.

Maclntyre, N. R. (2009). Spirometry for the Diagnosis and Management. Respiratory Care, 54, 10501057.

Nahas, M. (2013). Atividade física, saúde e qualidade de vida: conceitos e sugestões para um estilo de vida ativo. Brasil: Midiograf. 
Nakayama, S. (1990). New method for hydrotherapy and effects of flowwater training sytem. The Journal of Japonese Physical Therapy Associaton, 17, 211-215.

Neto, C., Cordovil, R., \& Lopes, F. (2015). Children's (in)dependent mobility in Portugal. Journal of Science and Medicine in Sport, 18, 299-303. https://doi.org/10.1016/j.jsams.2014.04.013

Sharifi, G., Mazreno, A. B., Salmani, I., \& Abyar, N. (2014). Effects of a Period of Selected Activity on Lung Capacities in Children 5-10 Years with Asthma Caused by Exercise. Internacional Journal of Pediatrics, 2, 13-16.

Stella, S., Vilar, A., Lacroix, C., Fisberg, M., Santos, R., Mello, M., \& Tufik, S. (2005). Efects of type of physical exercice and leisure activities on the depression scores of obese Brasilian adolescent girls. Brasilian Journal of Medical and Biological Research, v.38, nr11, pp: 1683-1689. Brasil.

Paulo, R. (2014). Efeitos da Atividade Física na Composição Corporal e nos Parâmetros Fisiológicos com Impacto no Estado de Saúde, de Alunos do Ensino Superior. Dissertação de Doutoramento, Universidade da Beira Interior, Covilhã, Portugal.

Troiano, R., Berringan, D., Dodd, K., Mâsse, L., Tilert, T., \& McDowell, M. (2008). Physical activity in the United States measured by accelerometer. Medicine \& Science In Sports \& Exercice, 40, 181-188. https://doi.org/10.1249/mss.0b013e31815a51b3

Wilder, P., \& Brennam, D. (1993). A standard measure for exercise prescription for aqua running. The American Journal of Sports Medicine, 21, 45-48. https://doi.org/10.1177/036354659302100108

\section{(9) $(\mathbb{0} \Theta \Theta$}

This work is licensed under a Attribution-NonCommercial-NoDerivatives 4.0 International (CC BY-NC-ND 4.0). 